\title{
New Aspects for Modernization Global Maritime Distress and Safety System (GMDSS)
}

\author{
M. Ilcev \\ Durban University of Technology, Durban, South Africa
}

\begin{abstract}
This paper describes the new aspects of modernization Global Maritime Distress and Safety System (GMDSS) proposing new developments and integrations of Radio and Satellite Communication, Navigation and Surveillance (CNS) and Global Ship Tracking (GST) systems in the function of enhanced Safety at Sea (SAS) and improved Search and Rescue (SAR) operations. To provide reliable tracking and detecting facilities of ships in distress have to be integrated the current Maritime Radio Communications (MRC) and Maritime Satellite Communications (MSC) systems with new Space Communication, Navigation and Surveillance Systems (CNS). The preliminary modernization aspects and plan for the GMDSS radio and satellite network are introduced in this paper. Based on the current state of the GMDSS and considerations on updating, upgrading and improving the system, some normative and technical aspects of the GMDSS modernization proposals are given. Further proposals on the GMDSS modernization plan were also considered.
\end{abstract}

\section{INTRODUCTION}

Since the invention of radio at the end of the 19th century, ocean going ships at sea have relied on Morse code, invented by Samuel Morse and first used in 1844, for distress and safety telecommunications, and on radio transceiver invented in early 1895 by Russian professor of physics Aleksandar Stepanovich Popov. The need for ship and coast radio stations to have and use radiotelegraph equipment, and to listen to a common radio frequency for Morse encoded distress calls via onboard radio station designed by Marconi, was recognized after the sinking of the liner Titanic in the North Atlantic in 1912.

Morse encoded distress calling has saved thousands of lives since its inception a century ago, but its use requires skilled radio operators spending many hours listening to the radio distress frequency. Its range on the Medium Frequency (MF) distress band of $500 \mathrm{kHz}$ is limited, and the amount of traffic Morse signals can carry is also limited. The SOS distress call was transmitted on dedicated radiotelegraphy band of $500 \mathrm{kHz}$, and the voice distress call Mayday was conducted on radiotelephony bands of $2182 \mathrm{kHz}$.

The maritime radio systems for commercial and distress communications worked very successfully for several decades and after invention of maritime satellite communication by the US Marisat in 1976 with only three satellites and ocean networks that are providing Maritime Satellite Service (MSS) in the Atlantic, Pacific and Indian Ocean areas. By the end of the 1980s, maritime satellite communication services at sea developed by Inmarsat had started to take an increasingly large share of the market for ship-to-shore communications.

For these reasons, the International Maritime Organization (IMO), a United Nations (UN) agency 
specializing in safety and security of shipping and preventing ships from polluting the seas, began looking at ways of improving maritime distress and safety communications. In 1979, a group of experts worldwide drafted the International Convention on Maritime Search and Rescue (SAR), which called for development of a global SAR plan.

Soon after, this group also passed a resolution calling for development by IMO of an GMDSS to provide the communication support needed to implement the SAR plan more effective. This new SAR system, which the world's maritime nations were implemented, is based upon a combination of radio and satellite services, and has changed international distress communications from being primarily ship-to-ship based to ship-to-shore Rescue Coordination Center (RCC) based. It spelled the end of Morse code communications for all but a few users, such as amateur radio operators and some military is still using this service.

The GMDSS provides for automatic distress alerting and locating in cases where a radio operator doesn't have time to send an SOS or MAYDAY call, and, for the first time, requires ships to receive broadcasts of Maritime Safety Information (MSI) to prevent a distress from happening in the first place. In 1988, IMO amended the Safety of Life at Sea (SOLAS) Convention, requiring ships to fit GMDSS equipment. Such ships were required to carry NAVTEX and satellite Emergency Position Indicating Radio Beacon (EPIRB) units by 1 August 1993, and had to fit all other GMDSS equipment by 1 February 1999.

\section{CURRENT GMDSS NETWORK}

The GMDSS plan was adopted by the International Maritime Organization (IMO) as part of the 1988 amendments to the 1974 International Convention for the SOLAS-74 and that the system was finally fully implemented in 1999. The GMDSS architecture ensures that a ship in distress and emergency situation anywhere in the world's oceans must always be heard and able to provide an answer. The GMDSS network with space and ground infrastructures provides for users (ships) and covers a unique combination of international technical and operational standards, recommendations and also includes globally coordinated use of radio frequencies at both ship and coast stations. The GMDSS network works well in the maritime industry and serves faithfully for seafarers onboard ships, but some of the technologies used in the system do not reveal their full potential and some functions can be performed using more modern technologies.

Taking into account the above statements, as well as the e-Navigation project, at the 86th session of the Maritime Safety Committee (MSC ${ }^{*}$ ), it was decided to include the sub-item "Analytical research to establish the need to review the elements and procedures of the GMDSS" in the work program of the IMO Subcommittee on Radio Communication and Search and Rescue (COMSAR Subcommittee). As a result of this work, in 2012, MSC ${ }^{*}$ approved a new unplanned exit of the GMDSS Review and Modernization Project. The project includes a review of High Level Review (completed in 2014), Detailed Review (completed in 2016), and the Modernization Plan (launched in 2016), based on data from previous work. The COMSAR Subcommittee was entrusted with being the coordinating body in this matter. At the end of 2013, after making changes to the organization of work of the IMO subcommittees, this issue falls into the competence of the new subcommittee on Navigation, Radio Communication and Search and Rescue (NSPS).

The GMDSS network is an integrated system consisting traditional Maritime Radio Communications (MRC) and new Maritime Satellite Communications (MSC), such as Inmarsat and Cospas-Sarsat subsystems. This integration has to ensure that no matter where a ship is in distress alert and aid can be dispatched. It also ensures the provision of Maritime Safety Information (MSI), both meteorological and navigational information, on a global basis at sea. The regulation governing the GMDSS network is contained in the International Convention for the Safety of Life at Sea (SOLAS) in 1974. The GMDSS requirements are contained in Chapter IV of SOLAS on Radiocommunications and were adopted in 1988.

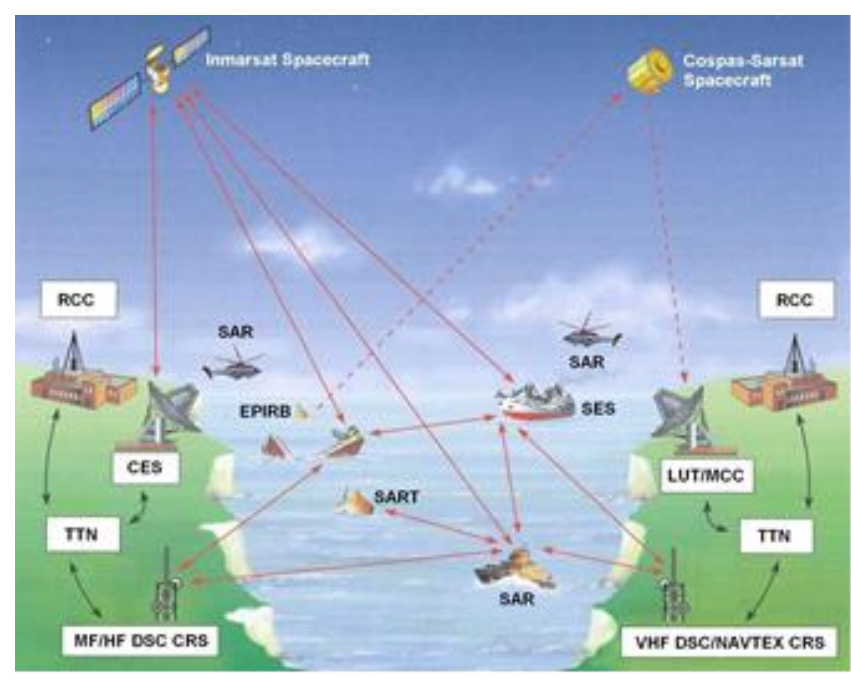

Figure 1. Current GMDSS Network

In fact, the GMDSS communications system under SOLAS complements the International Convention on SAR in 1979 was adopted to develop a global SAR plan, which current GMDSS network is illustrated in Figure 1. As already stated, this network is containing two main subsystems such as Radio and Satellite networks.

The traditional radio system is providing Very High Frequency (VHF), the Medium Frequency (MF) and High Frequency (HF) networks via Coast Radio Stations (CRS). While, Ships Radio Stations (SRS) consists onboard ship equipment terminals, such as Digital Selective Call (DSC), NAVTEX and Search and rescue radar Transponders (SART).

The Inmarsat Network is providing service for Ship Earth Stations (SES) via Geostationary Earth Orbit (GEO) Inmarsat satellites, and Terrestrial Telecommunication Network (TTN) and Mission Control Centres (MCC) and RCC to SAR forces. The 
RCC station receives distress signals and via SAR ships and helicopters give assistance to ships in distress. The Cospas-Sarsat Network is providing transmission of EPIRB distress signal via LEOSAR, GEOSAR and MEOSAR satellites, LUT stations and MCC, TTN and RCC to SAR forces.

\section{MODERNIZATION AND IMPROVEMENTS OF THE GMDSS SEA OPERATIONAL AREAS}

The novel deigns of global Satellite CNS subsystem and maritime GMDSS equipment include Global Ship Tracking (GST) as enhanced Long Range Identification and Tracking (LRIT), Satellite AIS (SAIS), Satellite Data Link (SDL), Satellite Automatic Dependent Surveillance - Broadcast (SADS-B) and Global Navigation Satellite System (GNSS) Augmentation SDL (GASDL) to benefit for improved alerts and SAR operation of ships in distress.

Due to the different radio and satellite communication systems incorporated into the GMDSS network having individual coverage limitations with respect to range and service provided, the equipment required to be carried by a ship is determined by the ship's area of operation, rather than by its size. The GMDSS network has divided the world's oceans into four distinct areas. The current requirements of GMDSS recommendations and regulations all GMDSS ships are required to carry onboard equipment appropriate to the sea area or areas coverage in which they operate, such as the following operational areas:

1 Sea Area A1 - This are is within the radiotelephone coverage of at least one VHF coast station in which continuous VHF Radio DSC alerting is available in area of approximately of 20$30 \mathrm{~nm}$ range. As stated in abstract, according to the new technologies and developments in this sea GMDSS area can be included Radio Ship Tracking (RST), VHD Data Link (VDL) similar to aeronautical VDL Mode 4 or new proposed VHF Data Exchange System (VDES),

Radio-Automatic Identification System (R-AIS) known as VHF AIS, Radio Automatic Dependent Surveillance-Broadcast (RADS-B), GNSS (GPS or GLONASS) Augmentation VDL-Broadcast (GAVDL-B);

2 Sea Area A2 - This area is within the radiotelephone coverage of at least one MF coast station in which continuous MF Radio DSC alerting is available in sea area of approximately $100 \mathrm{~nm}$ range (excluding Sea Area A1). However, in this area can be used VDL Mode-4 or VDES, RADS-B and Inmarsat mini-C or Inmarsat-C service;

3 Sea Area A3 - This large area is within the coverage area of an Inmarsat GEO satellite constellation in which continuous alerting is available in sea area of approximately between 76o $\mathrm{N}$ and 76o $\mathrm{S}$ range, (excluding Sea Areas A1 and A2). Besides, in this area can be used Global Ship Tracking (GST) proposed by the CNS Systems company from Durban, Satellite Data Link (SDL), Satellite Automatic Dependent Surveillance Broadcast (SADS-B), GNSS Augmentation SDL
(GASDL), Long Range Identification and Tracking (LRIT), Orbcomm Satellite-Automatic Identification System (S-AIS) and HF Data Link (HDL). Therefore, except Orbcomm S-AIS and HDL, all above stated satellite communication solutions can use Inmarsat GEO and Iridium Big Low Earth Orbit (LEO) satellite constellation;

4 Sea Area A4 - This is the remaining sea coverage outside areas A1, A2 and A3 (basically in the range of the Polar Regions). In both polar areas can be used Iridium satellite constellation employing equipment such as Iridium GST, SDL and SADS-B and as well as Orbcomm S-AIS and HDL.

In this case, there are discussions at IMO that may result in redefining Sea Areas A3 and A4 based on satellite coverage of systems other than Inmarsat, if such systems are approved for use within the GMDSS. This is not expected to come into force for several years.

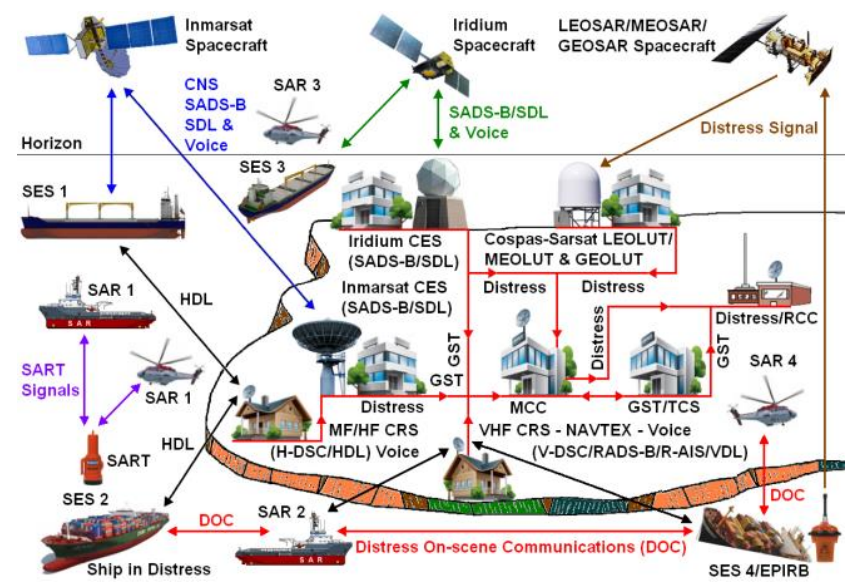

Figure 2. New Concept of GMDSS Network

However, the new concept of enhanced GMDSS space and ground network is developed and designed by CNS Systems Company from Durban, South Africa, which integrated system architecture, is illustrated in Figure 2. The main integrated components of enhanced GMDSS network are Radio VHF and MF/HF subsystem, Inmarsat GEO and Iridium Big LEO satellite communication subsystems and Cospas-Sarsat LEOSAR, MEOSAR and GEOSAR satellite subsystems. Radio system provides the same service via VHF and MF/HF bands, such as DSC and NAVTEX transmissions, including all applications listed in Sea Area A1 and same in A2, Inmarsat satellite network provides all service listed in A2 and A3, while and Iridium satellite network provides service listed in A4.

In such a way, new concept of GMDSS satellite system will provide Inmarsat and three Cospas-Sarsat subsystems including new proposals for Iridium. On the other hand, the LEO Orbcomm and Medium Erath Orbit (MEO) O3b networks, including the best hybrid constellation of GEO and Highly Elliptical Orbit (HEO) Molniya networks. 
4 DEVELOPING SCOPE FOR PREMODERNIZATION OF THE GMDSS NETWORK

The preliminary modernization plan of the GMDSS network is prepared by IMO, Inmrsat, Cospas-Sarsat and other participants is known as Work Program of the Modernization, which is consisting the following main components: General provisions; Functional requirements in accordance with the International Telecommunications Union (ITU) Radio Regulatio-ns (RR) and other documents of the ITU-R; The provision of satellite services to the GMDSS and the redefinition of the sea area A3; VHF Data Exchange System (VDES) or known as VDL; Navigation Data (NAVDAT); Routing distress signals and related information; Search and Rescue (SAR) technologies; High Frequency (HF) Data Link (HDL) and radio communication; Requirements for the transportation of GMDSS racks; False alerts; Training; Outdated provisions; and Explanations.

In the process of modernization of the GMDSS, the following provisions should be taken into account of the IMO Subcommittee on Navigation, Radio Communication and Search and Rescue (NCSR) [NCSR 3, 2016]:

1 Modernization Process - This process and including new and revised documents, should not exclude vessels that are not parties to the SOLAS Convention from any technical and economic reasons. The documentation and equipment intended for such vessels must fully comply with the GMDSS system;

2 Communications Statements - These systems of the IMO to the radiocommunication sector of the ITU should be guided by the principle that ships not party to the SOLAS Convention can use the GMDSS and the integrity of the system should be preserved in this case. Including, if necessary, ITU-R recommendations prescribed for these vessels the equipment and use of the GMDSS frequencies;

3 E-navigation System - The GMDSS modernization project should continue to support the needs of the new E-navigation strategy; and

4 New Technologies - In order to note the effectiveness of new technologies, as well as the compliance with the set goals, the human factor, in the process of modernization the GMDSS will be involved both onboard ships and at shore infrastructures.

Taking into account the above components of the IMO GMDSS Modernization Plan and a special work program were adopted that included the revision and development of regulatory documents, standards and also reference materials. Thus, the following list is presenting the necessary actions considered at the session of the NCSR subcommittee [NCSR 4/12, 2016], of the IMO coordinated work plan for the modernization project:

1 In 2018 - The NCSR subcommittee is completing the development of a Modernization Plan First draft amendments to the IMO SOLAS Convention and related documents and finalizing draft revision of the criteria for the provision of mobile satellite services;

2 In 2019 - Second draft amendments to the SOLAS Convention and related documents are provided together with testing the draft revision of the criteria for the provision of mobile satellite services in the GMDSS network;

3 In 2020 - Final draft amendments to the SOLAS Convention and related documents and draft functional requirements for NAVDAT have to be are performed;

4 In 2021 - Approval of amendments to the SOLAS Convention with related technical documents and as well as testing functional requirements for NAVDAT facilities have to be resolved;

5 In 2022 - Adoption of amendments to the SOLAS Convention (and related documents, as appropriate) has to be prepared; and

6 In 2023/24 - All proposed amendments to the SOLAS Convention have to enter into force in a due course.

\section{STANDARDIZATION OF THE PROBLEMS AND ASPECTS OF A MODERNIZATION PLAN}

The GMDSS network is integration complex of radio and satellite CNS systems, solutions and equipment intended to provide reliable emergency alert of any ship in distress situation, to ensure that any distress message will be received by shore or other ships in the immediate vicinity, to determine position of ship in distress, engage SAR operations with improved means of locating survivors and arrange prompt saving of their lives. The GMDSS concept was developed trough the IMO and other contributors to change the way for conducting maritime distress communications for enhanced SAR operations. The GMDSS solutions and equipment are mandatory for all ships subject to the SOLAS convention, which include cargo ships of 300 gross tones or greater and all passenger vessels on international voyages.

The GMDSS network was developed to modernize and enhance previous emergency ships radiocommunication system and to provide a more effective distress alerting by using DSC and other radio VHF, MF and HF equipment and backed up with new satellite techniques and technologies. In fact GMDSS uses various types of radio and satellite equipment to transmit and receive accurate and real distress signals and also to improve rescue communications, coordination and increases the following tasks:

1 Ability to locate survivors from ship in distress and including from aircraft landed at sea;

2 Likelihood that distress alert will be received by shore radio and satellite stations or other ships in vicinity;

3 Probability that an alert will be sent when a vessel is in a real distress and not false alert;

4 Capacity to provide mariners with vital Maritime Safety Information (MSI);

5 It is necessary to define the terms "Security messages" and "Other messages " and as well as state the requirements for radio installations to perform the above functions;

6 Provide the more reliable and effective types of GMDSS shipborne radio equipment to transmit and receive accurate and reliable distress signals; and 
7 Modern tracking systems for maritime applications are developed in high level, but still there are problems of reliability of existing tracking networks and equipment respectively. Several problems of tracking system for maritime applications can be further emphasized.

\section{TECHNICAL ASPECTS OF THE MODERNIZATION PLAN}

The more critical scenario for distress alerting situations is when ships are sailing across oceans and unfriendly coastal areas in very bad weather and sea conditions, when visible and audibility is almost zero, when sometimes technically is not possible to use surveillance radar and when is very difficult to detect surrounded ships for collision avoidance.

In order to ensure a more reliable, accessible and efficient situation on board in the event of possible awareness of distress, it will be necessary to use all new networks and monitoring and detection equipment, duchs as VHF-band Data Exchange System (VDES), Global Ships Tracking (GST) and GNSS Augmentation Satellite Data Link (GASDL).

\subsection{VHF-band Data Exchange System (VDES)}

The new VHF-band Data Exchange System (VDES) was developed by the International Association of Maritine Aids to Navigation and Lighthouse Authorities (IALA) to address the emerging signs of data transmission channel overload in the AIS (VDL) band and at the same time provides more wide and unhindered data exchange for the maritime community. The initial concept of VDES includes the function of an AIS (R-AIS), Application Specific Messages (ASM), VHF terrestrial and satellite communication segments.

The VDES network is one of the potential elements of E-navigation, which will exchange ASM transmission, thereby arranging operation of numerous applications to ensure safety, efficiency and protection of shipping, as well as environmental protection. In the future, the VDES network will have a significant positive impact on MSI network including Navigation Assistance Services (NAS) and Vessel Traffic Management System (VTMS).

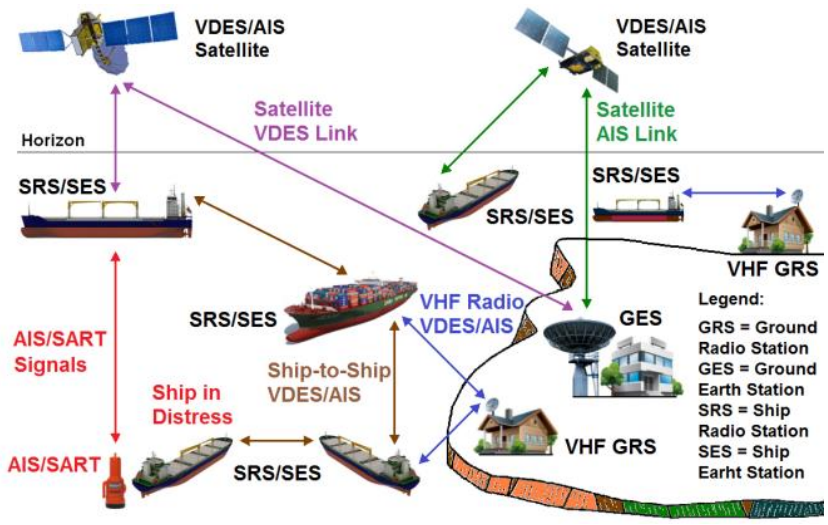

Figure 3. New Concept of VDES/AIS Network
In Figure 3 is illustrated the new concept of integrated VDES/AIS Network, which consists VDES/AIS space segment, ground segment integrated with GES and VHF GRS terminals and users segment containing SRS/SES terminals and AIS/SART beacons. The VDES/AIS Network provides satellite and VHF radio VDES/AIS links, inter-ship (ship-to-chip) communications, and AIS/SART signals. Thus, the AIS signals can be received by the R-AIS receiver and SART signals can be received by the onboard ships radars.

Using VDES can potentially provide a local VTMS, however VDES can also inlude concept deploying a space (satellite) segment for global coverage. The space segment of the system can be used for VTMS transmission in remote areas [Recommendation ITUR M.2092-0, 2015]. An insufficient study and proposals of the issue of sharing and comparability between the new developed satellite segment of the VDE system and the existing services in the same and adjacent frequency ranges caused the operating frequency range to not be determined at the ITU World Radiocommunication Conference in 2015 (WRC-2015) .

As a result, VDES as a whole is still not a complete functional system. As part of the 2015 IGC, the ITU approved the standard for VDES in the form of Recommendation ITU-R M.2092-0 [PP, 2015]. The unresolved issue of the approval of the satellite segment for data exchange channels in the VHF band (VDE). The approval of this issue is one of the goals of the 2019 World Radiocommunication Conference (WRC-2019).

The study of the vacant frequency ranges 156.0125-157.4375 MHz and 160.6125-162.0375 MHz will mainly concern interaction with existing mobile services, primarily for land and sea mobile services, as well as services within adjacent lower (from 154 $\mathrm{MHz}$ up to $156 \mathrm{MHz}$ ) and high (from $162 \mathrm{MHz}$ to 164 $\mathrm{MHz}$ ) frequency ranges. The concept of a VDES network will be developed under agenda item 1.9.2 at WRC-19:

1 Amendments to the ITU Radio Regulations (RR), including new spectrum allocations to the maritime mobile-satellite service (Earth-to-space and space-to-Earth), preferably in the frequency bands 156.0125-157.4375 $\mathrm{MHz}$ and 160.6125$162.0375 \mathrm{MHz}$ of Appendix 18, to create conditions for the operation of the VDES while ensuring that this segment does not impair the operation of existing VDES terrestrial segments, ASM, AIS network and does not impose any additional restrictions on existing services in these and adjacent frequency bands referred to in d) and e) of the section, recognizing ITU Resolution 360 (Rev. WRC-15).

2 In addition to other applications, the use of VDES must be considered in all kinds of future VTMS dissemination mechanisms.

\subsection{Global Ship Tracking (GST) Network}

The proposed GST network and onboard equipment, as the best solution for modernization GMDSS, can be any airborne GPS/Iridium or GPS/Inmarsat equipment installed together with GPS/Iridium or 
GPS/Inmarsat antenna secretly onboard ship. Because these devices are not very expensive can be installed two onboard ships or one as a back up. In fact, the GST mission can use existing GNSS satellite network for receiving GNSS signals, a real global Iridium Big Low Earth Orbit (LEO) satellite network with intersatellite links or a near-global Inmarsat GEO satellite network for commercial and distress data communications.

There are specific shipborne technologies that were designed for special purposes and they are great at providing the intended service for the dedicated solution. For instance AIS is a good global generalized picture of assets in the loosest terms possible because it's not what it was built for. There are applications where AIS is good to great, safety while sailing at sea, not getting ran over by a cargo ship at night or low visibility, etc. But oceangoing ships need discrete and autonomous systems coming up that are capable to replace medium to high intelligence satellite tracking devices such as Global Ship Tracking (GST), which architecture is illustrated in Figure 4.

The new GST network has to provide special Maritime Information Service (MIS) for positioning data of ships in certain sailing ocean area. Namely, this service will be an automatically messaging system of Position, Velocity and Time (PVT) data of autonomous onboard ships unit containing GPS receiver, satellite transceiver and own battery power supply in combination with main ship power supply. The PVT data will be sent in certain intervals via Inmarsat or Iridium satellite, Coast Earth Stations (CES), Internet network to the special and independent Tracking Control Stations (TCS). Thus, these TCS terminals will receive, process and store PVT data of all ship sailing in its ocean area and show on a special screen similar to the radar display. Each TCS terminal has to be directly connected to the MCC, RCC, Ship Traffic Control (STC) and Ship Traffic Management (STM) systems. In addition, for enhanced service of collision avoidance, all ships sailing in certain ocean area can request from TCS terminals position of all adjacent ships or they simply can provide polling position data of all adjacent ships.

In such a way, the TCS terminal can receive PVT data from any ship, process and display on radar like display. What LRIT cannot do, GST can provide determination and surveillance of all ships in certain sea area for enhanced collision avoidance, assist SAR to find in shortest time any missing ship, provide data for immediate detecting position of ship captured by pirates and it is able to improve GMDSS facilities.

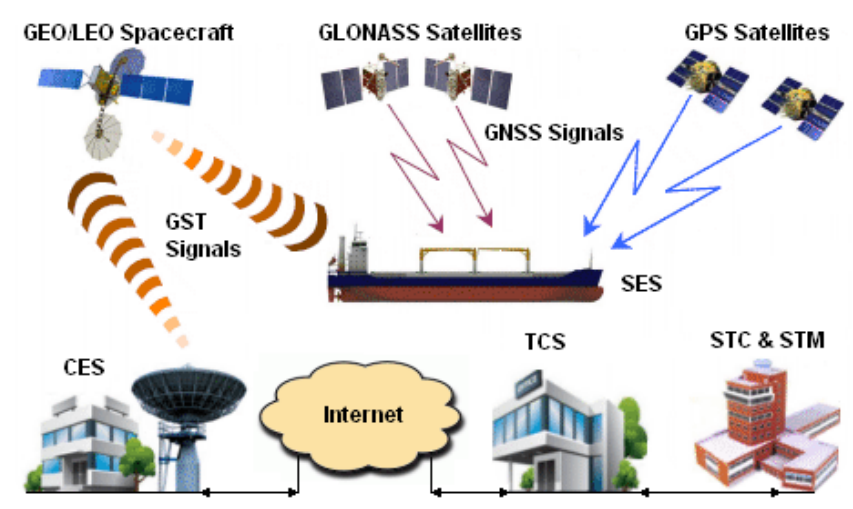

Figure 4. Maritime GASDL System for Enhanced GMDSS Network

\subsection{Maritime GNSS Augmentation Satellite Data Link (GASDL) Network}

The Regional Satellite Augmentation System (RSAS) network is a combination of ground monitoring and space communication infrastructures dedicated to provide augmentation of standard GPS or GLONASS signals, which diagram of GNSS Augmentation Satellite Data Link (GASDL) network is illustrated in Figure 5. The major functions being provided by RSAS are as follows: 1. Differential corrections are determined to improve GNSS signal accuracy; 2. Integrity monitoring is predisposed to ensure that errors are within tolerable limits with a very high probability and thus ensures safety; and 3. Ranging is proposed to improve availability.

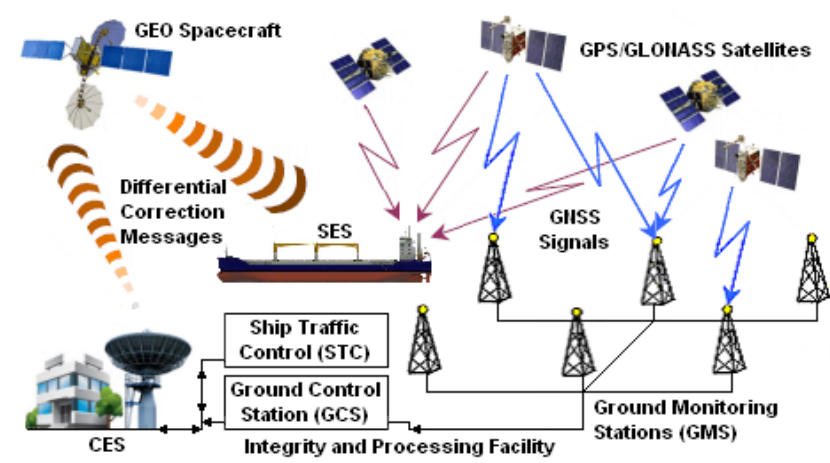

Figure 5. Maritime GASDL System for Enhanced GMDSS Network

In the same way, the numbers of Reference Stations (GMS) are receiving not augmented signals of GPS or GLONASS satellites, processing and forwarding this data to Master Station (GCS). The GCS terminals provide processing of GNSS data to determine the differential corrections and bounds on the residual errors for each monitored satellite and for each area. The GCS terminal is providing determination of the clock, ephemeris and ionospheric errors (ionospheric corrections are broadcast for selected area) affected during propagation. The corrections and integrity information from the GCS terminal are then sent to each RSAS Coast Earth Station (CES) and uplinked to the GEO Satellites.

Thus, these separate differential corrections are broadcast by RSAS CES through GEO satellite data 
link via GNSS transponder at the same frequency used by not augmented GPS receiver. For instance, augmented GPS Rx is receiving augmented signals of GPS satellite and determining more accurate position of ships. Not augmented GPS Rx can also receive augmented signals if is provided an adequate software or hardware. The most important stage in this network is to provide technical solution that augmented position of ships can be sent automatically via SDL or voice to CES and STC centre. Finally, these positioning signals can be processed by special processor and displayed on look like radar display, whish traffic controller is using for STC and management for enhanced ship traffic control and improved collision avoidance in certain monitoring sea area.

\section{NAVIGATION DATA (NAVDAT)}

An MF radio system is designed for use in the maritime mobile service operating in the $500 \mathrm{kHz}$ band for digital broadcasting of information relating to maritime safety and security in the coast-to-ship direction.

The NAVDAT system uses a time slot allocation similar to the International Automated Alert System known as Navigational Telex (NAVTEX), which IMO can coordinate in the same way.

The NAVDAT system can also operate in Single Frequency Network (SFN) mode. In this case, the transmitters are synchronized in frequency, and the data for transmission should be the same for all transmitters. Thus, the digital NAVDAT $500 \mathrm{kHz}$ system provides broadcast transmission of any type of message in the shore-to-ship direction with encryption capability.

Any broadcast message must come from a secure and managed source. Types of messages for broadcast transmission include, but are not limited to, the following particulars: 1) Navigation safety; 2) Security Issues; 3) Data on piracy event; 4) SAR; 5) Meteorological reports; 6) Pilot or port communications; and 7) File transfer of the ship traffic system.

These messages broadcast information for vessels, groups of vessels, or in certain areas of navigation. Besides, these messages can be addressed to a single vessel using the Maritime Mobile Service Identity (MMSI). The organization of the NAVDAT system is determined by five factors that ensure the performance of the following functions [Text. ITU-R M.2010, 2012]:

1 System of Information and Management (SIM) Collects all types of information and manages this information, creates message files to be transmitted and creates transmission programs in accordance with the priority of message files and the needs of the replay;

2 Coastal Network - Provides transportation of message files from sources to transmitters;

3 Shore Transmitter - Accepts message files from SIM, converts message files to a signal with Orthogonal Frequency Division Multiplexing
(OFDM) and transmits an RF signal to the antenna for broadcast to ships;

4 Transmission Channel - Transmits radio frequency signals at $500 \mathrm{kHz}$; and

5 Ship Receiver - Receive and demodulates an RF signal with OFDM, restores message files and sorts message files and makes them available to the target equipment in accordance with the application of the message files.

\section{NORMATIVE ASPECT OF A MODERNIZATION PLAN}

Marine safety requirements are provided in chapter XI-2 of the IMO SOLAS Convention. The Ship Security Alert System (SSAS) does not imply the establishment of radio communications with other ships or coastal radio stations, and therefore, security messages are not part of the ship-to-ship and ship-toshore communications. These messages are directly addressed to the competent authorities. It follows from the above that security messages should not be a functional requirement for the GMDSS network, however, chapter IV should oblige vessels to be able to provide security-related communications and give a clear definition "Security related communications". Therefore, it is proposed to add to definition IV/2 the definition that "Security related messages" means messages associated with updating security levels, incidents or security threats, as well as security information prior to entry to the port.

At present, many maritime Coastal Radio Stations (CRS) terminals state-owned are providing public correspondence in most cases are closed, in contrast to the time when the GMDSS system was just in its infancy. However, the equipment providing this type of communication is still relevant. This type of communication is carried out through commercial services that are in no way associated with coastal radio stations, and the term "Private Messages" itself is no longer widely used. In this regard, for the GMDSS Modernized System, it is proposed to change the term "Private Messages" to "Other Messages" and include new features in this definition, but not as part of the functional requirements of the GMDSS. It is proposed to revise the term "General Radio Communication (GRC)" by agreeing it with the Radio Regulations. The Proposed new definition for GRC [NCSR 4/12, 2016] states: "General radio communication means the exchange of official messages, but not about distress, transmitted by radio".

\section{CONCLUSION}

Discussions on the modernization of the GMDSS network are ongoing. The future of the GMDSS Modernization Plan is closely connected with the development of the e-Navigation project, and it is important to note the role of radio systems in this process. Undoubtedly, the data network will become one of the most important parts of the e-Navigation and reliable tracking systems projects. When carrying 
out modernization of the GMDSS, it is necessary, firstly, to identify the needs of real users, and, secondly, to realize that the modernization of maritime radio communications should not be limited only by technical requirements. In addition to the above, it is necessary to provide a sufficient amount of a human-machine interface and human resources, including staff training.

When upgrading the system, all the "flaws" of the initial development and operation of the GMDSS network should be taken into account. In addition, the modernization process must be continuous and open, remain modern, and meet the expected requirements of electronic navigation. To ensure this, a mechanism for the continuous evolution of GMDSS should be created on a systematic basis. With this approach to the modernization of the GMDSS, it is very important that the integrity of the system is not violated.

\section{REFERENCES}

[1] IMO, "International Convention for the Safety of Life at Sea (SOLAS)", London, UK, 2014.

[2] ITU, "Radio Regulations", Geneva, Switzerland, 2016.

[3] IMO, "IMO Subcommittee on Navigation, Radiocommunications and Search and Rescue -NPSA 3", Report of the MSC, London, UK, 2016.

[4] Ilcev D.S., "Global Mobile Satellite Communications for Maritime, Land and Aeronautical Applications Volume 2", Springer, Boston, US, 2017.

[5] IMO, "IMO Subcommittee on Navigation, Radiocommunications and Search and Rescue -NPSA 3", Report of the MSC, London, UK, 2016.

[6] Maini A.K. \& Agrawal V., "Satellite Technology Principles and Applications", Wiley, Chichester, UK, 2007.
[7] IMO, "IMO Subcommittee on Navigation, Radiocommunications and Search and Rescue -NPSA 3", Report of the MSC, London, UK, 2016.

[8] Calcutt D. \& Tetley L., "Understanding GMDSS", Edward Arnold, London, 1994.

[9] Calcutt D. \& Tetley L., "Satellite Communications, Principles and Applications", Elsevier, Oxford, 2004.

[10] Pratt S.R. at all, "Operational and Performance Overview of the Iridium Low Earth Orbit Satellite System", IEEE Communications Surveys, New York, US, 1999

[11] Kulakov K., "The point of view on the modernization plan for GMDSS", Admiral Ushakov Maritime State University, Novorossiysk, Russia, 2019.

[12] Bauk S., "A Review of NAVDAT and VDES as Upgrades of Maritime Communication Systems", Advances in Maritime Navigation and Safety of Sea Transportation (pp. 82-87), TransNav, Vol. 11, No 1, Gdynia, Poland, 2019.

[13] IMO, "Global Maritime Distress and Safety System (GMDSS) Handbook", IMO, London, UK, 2001

[14] IMO, "Updating of the GMDSS Master Plan and Guidelines on MSI (Maritime Safety Information) Provisions", IMO, London, UK, 2019

[15] IMO, " Review and Modernization of the Global Maritime Distress and Safety System (GMDSS)", IMO, London, UK, 2014.

[16] ITU, "Technical specifications for the VHF data interchange system in the VHF maritime mobile band", Geneva, Switzerland, 2015.

[17] IMO, " Review and Modernization of the Global Maritime Distress and Safety System (GMDSS)", IMO, London, UK, 2014.

[18] IMO, "Review and Modernization of the GMDSS", IMO, London, UK, 2012.

[19] ITU, "Adaptive radio systems at frequencies below about $30 \mathrm{MHz}$ ", Geneva, Switzerland, 2003.

[20] IMO, "Harmonization of GMDSS Requirements for Radio Installations on board SOLAS Ships", IMO, London, IMO, UK, 2004.

[21] AMSA, "Australian Global Maritime Distress and Safety System (GMDSS) Handbook", AMSA, Camberra, Australia, 2013. 